\title{
Effect of Maternal Nonalcoholic Fatty Liver Disease and Dietary Choline Status on Body Mass and Lipid Profile in Rat Offspring
}

\author{
Joanna Mikołajczyk-Stecyna $^{1}$, Ewelina Żuk ${ }^{1}$, Krzysztof Olszyński ${ }^{2}$, Piotr Celichowski ${ }^{3}$, \\ Marcin Ruciński ${ }^{3}$ and Agata Chmurzynska ${ }^{1}$ \\ ${ }^{1}$ Poznan University of Life Sciences, Institute of Human Nutrition and Dietetics, Poznan, Poland, \\ ${ }^{2}$ M. Mossakowski Medical Research Centre, Polish Academy of Sciences, Behavior and Metabolism Research \\ Laboratory, Warsaw, Poland and \\ ${ }^{3}$ Poznan University of Medical Sciences, Department of Histology and Embryology, Poznan, Poland
}

\section{Abstract}

Both maternal metabolic status and nutrition during pregnancy and lactation may have a programming effect on offspring metabolism. The aim of this study was to examine the role of the dietary choline supply during pregnancy and lactation in rat dams suffering from nonalcoholic fatty liver disease (NAFLD) on body weight and plasma lipid profile of the progeny.

The research protocol was approved by the local ethics committee. The study groups included the offspring of 1 . healthy dams receiving choline during pregnancy and lactation (the control group); 2. NAFLD dams receiving choline during pregnancy and lactation (NN); 3. NAFLD dams receiving choline during pregnancy and a choline-deficient diet during lactation (ND); 4. NAFLD dams receiving a choline-deficient diet during pregnancy and a supply of choline during lactation (DN); and 5. NAFLD dams receiving a choline-deficient diet during both pregnancy and lactation (DD). Body mass and plasma lipid profile were assessed in male and female rats from each group on day 3 (3d), day 24 (24d), and day 90 (90d).

Body mass was significantly lower in the male offspring of the DD and DN groups than in the control group. Differences were observed at all times $(3 \mathrm{~d}: \mathrm{p}=0.0023 ; 24 \mathrm{~d}: \mathrm{p}<0.0001 ; 90 \mathrm{~d}: \mathrm{p}<0.0001)$. Moreover, body mass was significantly higher in the male offspring of the control group than in any other group. In the female progeny, body mass was higher in the control group than in the ND (24d: $\mathrm{p}<0.0001 ; 90 \mathrm{~d}: \mathrm{p}=0.0067)$ or NN (24d: $\mathrm{p}=0.0058)$ groups.

Total plasma cholesterol concentration was higher in the $90 \mathrm{~d}$ males of the control group than in the DD group ( $\mathrm{p}=0.0163)$ and in the $24 \mathrm{~d}$ females of the NN group than in the ND group $(p=0.0495)$. In the $3 \mathrm{~d}$ animals, LDL was higher $(\mathrm{p}=0.0083)$ but HDL was lower $(p=0.0196)$ in male rats of the DD and DN groups than in the NN and ND groups. Neither age nor sex affected LDL levels. The plasma levels of triglycerides were not affected by the dietary regimen, sex, or age of the animals.

Maternal NAFLD and dietary choline status during pregnancy and lactation affect body mass and lipid profile in rat offspring, and the effects of maternal programming are more pronounced in male offspring than in female.

The project was financed by the National Science Centre, Poland (2016/21/D/NZ9/00360).

\section{Conflict of Interest}

There is no conflict of interest 